\title{
Behaviour of boundary functions for quantum billiards
}

\author{
A Bäcker ${ }^{1}$, S Fürstberger ${ }^{1}$, R Schubert $^{2}$ and F Steiner $^{1}$ \\ ${ }^{1}$ Abteilung Theoretische Physik, Universität Ulm, Albert-Einstein-Allee 11, D-89069 Ulm, \\ Germany \\ ${ }^{2}$ Service de Physique Théorique, CEA/DSM/SPhT, Unité de recherche associée au CNRS, \\ CEA/Saclay, F-91191 Gif-sur-Yvette Cedex, France \\ E-mail: arnd.baecker@physik.uni-ulm.de, silke.fuerstberger@physik.uni-ulm.de, \\ schubert@spht.saclay.cea.fr and frank.steiner@physik.uni-ulm.de
}

Received 19 July 2002

Published 19 November 2002

Online at stacks.iop.org/JPhysA/35/10293

\begin{abstract}
We study the behaviour of the normal derivative of eigenfunctions of the Helmholtz equation inside billiards with Dirichlet boundary condition. These boundary functions are of particular importance because they uniquely determine the eigenfunctions inside the billiard and also other physical quantities of interest. Therefore, they form a reduced representation of the quantum system, analogous to the Poincaré section of the classical system. For the normal derivatives we introduce an equivalent to the standard Green function and derive an integral equation on the boundary. Based on this integral equation we compute the first two terms of the mean asymptotic behaviour of the boundary functions for large energies. The first term is universal and independent of the shape of the billiard. The second one is proportional to the curvature of the boundary. The asymptotic behaviour is compared with numerical results for the stadium billiard, different limaçon billiards and the circle billiard, and good agreement is found. Furthermore, we derive an asymptotic completeness relation for the boundary functions.
\end{abstract}

PACS numbers: $03.65 . \mathrm{Sq}, 05.45 . \mathrm{Mt}$

\section{Introduction}

The study of eigenfunctions of quantum systems, in particular with chaotic classical dynamics, has attracted a lot of attention. A prominent class of examples is provided by billiard systems, which classically are given by the free motion of a particle inside some domain with elastic 
reflections at the boundary. The corresponding quantum system is described by the Helmholtz equation inside a compact domain $\Omega \subset \mathrm{R}^{2}$ (in units $\hbar=1=2 \mathrm{~m}$ ),

$$
\Delta \psi_{n}(\boldsymbol{x})+k_{n}^{2} \psi_{n}(\boldsymbol{x})=0 \quad \boldsymbol{x} \in \Omega
$$

with (for example) Dirichlet boundary conditions

$$
\psi_{n}(\boldsymbol{x})=0 \quad \boldsymbol{x} \in \partial \Omega
$$

where the normalized eigenfunctions $\psi_{n}(\boldsymbol{x})$ are in $L^{2}(\Omega)$. A detailed knowledge about the behaviour of eigenvalues and the structure of eigenstates is relevant for applications, for example in microwave cavities or mesoscopic systems (see, e.g., [1], and references therein).

A particular nice feature of the classical dynamics in Euclidean billiards is the existence of a global Poincaré section based on the boundary $\partial \Omega$. This Poincaré section facilitates the study of the classical dynamics considerably, because the dynamics is reduced to an area-preserving map on the two-dimensional compact surface of section. It appears therefore desirable to look for a similar reduced representation of the quantum-mechanical problem. One representation is provided by the boundary integral method. The boundary integral method transforms the two-dimensional Helmholtz equation (1) for the eigenfunctions with Dirichlet boundary condition to a one-dimensional integral equation on the boundary $\partial \Omega$. This method involves the normal derivative of the eigenfunctions, hereafter called the boundary functions,

$$
u_{n}(s):=\left.\frac{\partial}{\partial n_{x}} \psi_{n}(\boldsymbol{x})\right|_{\boldsymbol{x}=x(s)} \equiv\left\langle\boldsymbol{n}(s), \nabla \psi_{n}(\boldsymbol{x}(s))\right\rangle
$$

where $\boldsymbol{x}(s)$ is a point on the boundary $\partial \Omega$, parametrized by the arclength $s$, and $\boldsymbol{n}(s)$ denotes the outer normal unit vector to $\partial \Omega$ at $\boldsymbol{x}(s)$. The integral equation for the boundary function is of the form

$$
u=-\boldsymbol{H}_{1}(k) u
$$

where $\boldsymbol{H}_{1}(k)$ is an integral operator depending on the parameter $k$ (for the explicit form of its integral kernel, see equation (25) below). This equation has solutions only for a discrete set of values of the parameter $k$ which, when real valued, give the eigenvalues of the Helmholtz equation. The associated solution $u$ is the normal derivative of the corresponding eigenfunction which can be obtained from $u$ via an integral formula using the free Green function on the plane.

This reduction to the boundary is very useful for the numerical computation of eigenvalues and eigenfunctions. The boundary function allows for a direct expression of the corresponding eigenfunction, its normalization [2], momentum distribution [3], autocorrelation function [4] and other quantities of interest. Furthermore, the boundary functions are the basis to define a Husimi representation of the eigenstates over the classical Poincaré section (see, e.g., $[5,6]$ ) and therefore provide a direct connection with the classical Poincaré map. This is in particular useful in situations where one is interested in fine structures of the eigenstates and their relation to the classical dynamics, as in the field of quantum chaos. From this it is clear that the boundary functions deserve a study in their own right. A profound knowledge of their properties can then be used to obtain a description for the above-mentioned quantities.

There are two main aspects concerning the boundary functions. First, one can consider these functions as a possible set of basis vectors that span a kind of natural space for the reduced quantum system. Here the question of orthogonality and completeness of the boundary functions arises. Second, as the boundary functions contain all information on the quantum system inside the given domain, it is very interesting to see how the properties of the eigenstates of the Helmholtz equation (1) are reflected in the boundary functions. The last point is our main interest in this paper where we study the mean semiclassical behaviour of the sequence of boundary functions in terms of a spectral average. 
A classical example of a spectral quantity is the spectral staircase function (integrated level density)

$$
N(k):=\#\left\{n \in N \mid k_{n} \leqslant k\right\}
$$

whose asymptotic behaviour for $k \rightarrow \infty$ is given by the Weyl formula (see [7])

$$
N(k)=\frac{\mathcal{A}}{4 \pi} k^{2}-\frac{\mathcal{L}}{4 \pi} k+o(k) .
$$

Here $\mathcal{A}$ denotes the area of the billiard and $\mathcal{L}$ the length of the boundary $\partial \Omega$. It is a well-known observation that the first two terms of (6) usually describe the mean behaviour of $N(k)$ very well, even down to the ground state (see, for example, figure 2 in [8]).

In the same way one can consider the sum over a sequence of the normalized eigenfunctions $\psi_{n}$ up to some given energy $k^{2}$,

$$
\Psi(k, \boldsymbol{x}):=\sum_{k_{n} \leqslant k}\left|\psi_{n}(\boldsymbol{x})\right|^{2} .
$$

For billiards with $C^{\infty}$-boundary ([9], theorem 17.5.10) implies

$$
\Psi(k, \boldsymbol{x})=\frac{1}{4 \pi} k^{2}-\frac{1}{4 \pi} \frac{J_{1}(2 d(\boldsymbol{x}) k)}{d(\boldsymbol{x})} k+R(k, \boldsymbol{x})
$$

where $d(\boldsymbol{x})$ is the distance of the point $\boldsymbol{x} \in \Omega$ to the boundary $\partial \Omega$, and $J_{1}(z)$ denotes the Bessel function. The remainder $R(k, x)$ satisfies the estimate $|R(k, x)| \leqslant C k$ for large $k$. The second term in (8) describes the influence of the boundary. This result is, as the Weyl formula for the mean behaviour of the spectral staircase function, completely independent of the classical chaoticity of the underlying system. So it applies equally well to integrable, mixed and chaotic systems. To illustrate the behaviour of $\Psi(k, \boldsymbol{x})$, let us consider a member of the family of limaçon billiards introduced by Robnik [10, 11], whose boundary is given in polar coordinates by $\rho(\varphi)=1+\varepsilon \cos \varphi$ where $\varepsilon \in[0,1]$ is the family parameter. In figure 1 we show a three-dimensional plot of $\Psi(k, x) / k^{2}$ for the desymmetrized limaçon billiard with parameter $\varepsilon=0.3$ using the first 100 eigenfunctions of odd symmetry (i.e. with Dirichlet boundary conditions on the symmetry axis). For this parameter value the classical billiard has a mixed phase space (see figure 1 in [12]). The agreement of the asymptotic behaviour given by (8) with $\Psi(k, \boldsymbol{x})$ is very good (see [13] for further examples).

In analogy, a similar behaviour for the sum over the sequence of boundary functions $u_{n}(s)$ is expected in the limit $k \rightarrow \infty$, i.e.

$$
\sum_{k_{n} \leqslant k} \frac{\left|u_{n}(s)\right|^{2}}{k_{n}^{2}} \sim \frac{1}{4 \pi} k^{2}+\cdots .
$$

In [14], it has been conjectured that the asymptotic behaviour of a similar sum, $\sum_{k_{n} \leqslant k}\left|u_{n}(s)\right|^{2}$, is $D_{1} k^{4}+D_{2} \kappa(s) k^{3}$, where $\kappa(s)$ is the curvature of the boundary at the point $s \in \partial \Omega$. In this work, we derive for the sum (9) the asymptotic behaviour $c_{1} k^{2}+c_{2} \kappa(s) k$ including the constants. The first constant turns out to be $c_{1}=\frac{1}{4 \pi}$ which is consistent with the leading term of the Weyl formula (6). Multiplying (9) by $\frac{1}{2}\langle\boldsymbol{n}(s), \boldsymbol{x}(s)\rangle$ and integrating over the billiard boundary gives $N(k)$ for the left-hand side, because of the following normalization relation for $u_{n}(s)$ [2] (for alternative derivations and more general boundary conditions, see [15, 16])

$$
\frac{1}{2} \int_{\partial \Omega}\langle\boldsymbol{n}(s), \boldsymbol{x}(s)\rangle\left|u_{n}(s)\right|^{2} \mathrm{~d} s=k_{n}^{2} .
$$

For the right-hand side one uses

$$
\frac{1}{2} \int_{\partial \Omega}\langle\boldsymbol{n}(s), \boldsymbol{x}(s)\rangle \mathrm{d} s=\mathcal{A}
$$



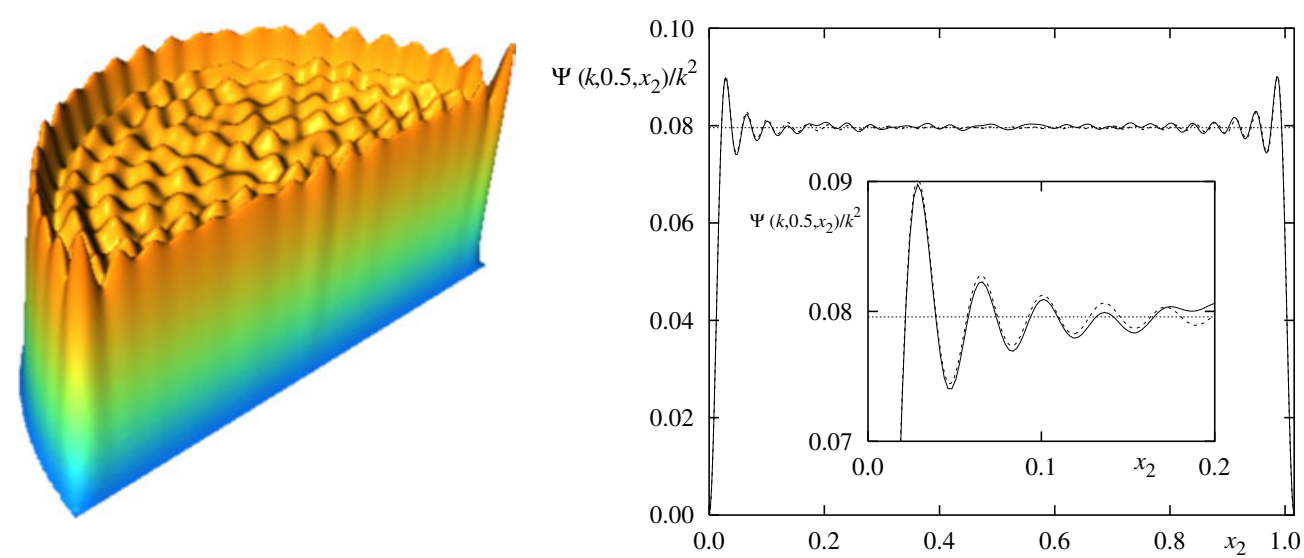

Figure 1. Three-dimensional plot of $\Psi(k, x) / k^{2}$ for the desymmetrized limaçon billiard for $\varepsilon=0.3$ and $k$ chosen such that $N(k)=100$. To the right a section of $\Psi(k, x) / k^{2}$ at $x_{1}=0.5$ for the case $N(k)=1000$ is shown and compared with the asymptotic result (8) (dashed curve). The horizontal dotted line shows the leading term $1 /(4 \pi)$. The inset shows a magnification.

to obtain the leading term of the Weyl formula (6). The next to leading term can be determined by using

$$
\int_{\partial \Omega} \kappa(s)\langle\boldsymbol{n}(s), \boldsymbol{x}(s)\rangle \mathrm{d} s=\mathcal{L}
$$

which for billiards with $C^{\infty}$-boundary follows from $\frac{\mathrm{d}}{\mathrm{d} s} \boldsymbol{t}(s)=-\kappa(s) \boldsymbol{n}(s)$ and partial integration (here $t(s)$ is the unit tangent vector at $s \in \partial \Omega$ ).

In the following, we derive the full asymptotic series for the sum in equation (9) and compute the first two terms explicitly. To this end, in section 2 an integral equation on the boundary is derived which is then used in section 3 to obtain the mean behaviour of the boundary functions. Some of the more technical details are given in the appendices. In section 4 , we provide a numerical test of the asymptotic behaviour at finite energies. We conclude with a short summary.

\section{An integral equation on the boundary}

Our main tool for the analysis of the semiclassical behaviour of the boundary functions is a boundary Green function which we define as

$$
\sum_{n \in \mathrm{N}} \frac{1}{k^{2}-k_{n}^{2}} u_{n}(s) u_{n}^{*}\left(s^{\prime}\right) .
$$

This is the analogue of the standard Green function inside the billiard, where we have replaced the eigenfunctions by their normal derivatives on the boundary. Our aim in this section is to derive an integral equation for (13), which can be solved recursively, and therefore leads to an expansion of (13) in terms of known functions. The method is very similar to the one used by Balian and Bloch [17], and our expansion on the boundary is the exact analogue of their multiple reflection expansion.

The energy-dependent Green function for the Dirichlet Laplacian on $\Omega$ is given by

$$
G(E, \boldsymbol{x}, \boldsymbol{y})=\sum_{n \in \mathrm{N}} \frac{\psi_{n}(\boldsymbol{x}) \psi_{n}^{*}(\boldsymbol{y})}{E-k_{n}^{2}}
$$


for $(\boldsymbol{x}, \boldsymbol{y}) \in \Omega \times \Omega$, where $k_{n}^{2}$ and $\psi_{n}$ are the eigenvalues and normalized eigenfunctions of $\Delta$, respectively, and $E$ is a complex parameter. This Green function is the unique solution of the equation

$$
\left(\Delta_{x}+E\right) G(E, \boldsymbol{x}, \boldsymbol{y})=\delta(\boldsymbol{x}-\boldsymbol{y})
$$

in $\Omega$ which satisfies Dirichlet boundary conditions. To obtain a similar relation on the boundary we define

$$
\begin{aligned}
g\left(k, s, s^{\prime}\right): & =\left.\frac{\partial}{\partial n_{x}} \frac{\partial}{\partial n_{y}} G\left(k^{2}, \boldsymbol{x}, \boldsymbol{y}\right)\right|_{\boldsymbol{x}=\boldsymbol{x}(s), \boldsymbol{y}=\boldsymbol{y}\left(s^{\prime}\right)} \\
& =\sum_{n \in \mathrm{N}} \frac{1}{k^{2}-k_{n}^{2}} u_{n}(s) u_{n}^{*}\left(s^{\prime}\right)
\end{aligned}
$$

where $k=\sqrt{E} \in \mathrm{C}, \operatorname{Im} k>0$, is the branch of the square root of $E$ which has positive imaginary part.

We will now derive an integral equation for $g\left(k, s, s^{\prime}\right)$. Let

$$
G_{0}(E, \boldsymbol{x}, \boldsymbol{y}):=\frac{1}{(2 \pi)^{2}} \int_{\mathrm{R}^{2}} \frac{1}{E-|\boldsymbol{\xi}|^{2}} \mathrm{e}^{\mathrm{i}\langle\boldsymbol{\xi}, \boldsymbol{x}-\boldsymbol{y}\rangle} \mathrm{d}^{2} \xi
$$

be a free Green function which satisfies $\left(\Delta_{x}+E\right) G_{0}(E, \boldsymbol{x}, \boldsymbol{y})=\delta(\boldsymbol{x}-\boldsymbol{y})$ on $\mathrm{R}^{2}$. This function depends holomorphically on $E$ in the cut plane $\mathrm{C} \backslash \mathrm{R}^{+}$. Additionally, we introduce the auxiliary functions $f\left(\boldsymbol{x}, s^{\prime}\right):=\left.\frac{\partial}{\partial n_{y}} G(E, \boldsymbol{x}, \boldsymbol{y})\right|_{\boldsymbol{y}=\boldsymbol{y}\left(s^{\prime}\right)}$ and $f_{0}\left(\boldsymbol{x}, s^{\prime}\right):=\left.\frac{\partial}{\partial n_{y}} G_{0}(E, \boldsymbol{x}, \boldsymbol{y})\right|_{\boldsymbol{y}=\boldsymbol{y}\left(s^{\prime}\right)}$. Then

$$
\left(\Delta_{x}+E\right)\left(f\left(\boldsymbol{x}, s^{\prime}\right)-f_{0}\left(\boldsymbol{x}, s^{\prime}\right)\right)=0
$$

for $\boldsymbol{x} \in \Omega \backslash \partial \Omega$ and therefore we can represent this difference as a single-layer potential

$$
f\left(\boldsymbol{x}, s^{\prime}\right)-f_{0}\left(\boldsymbol{x}, s^{\prime}\right)=S L_{\mu}\left(\boldsymbol{x}, s^{\prime}\right):=\int_{\partial \Omega} G_{0}(E, \boldsymbol{x}, \boldsymbol{y}(s)) \mu\left(s, s^{\prime}\right) \mathrm{d} s .
$$

The density $\mu\left(s, s^{\prime}\right)$ is determined by the boundary condition

$$
-f_{0}\left(\boldsymbol{x}\left(s^{\prime \prime}\right), s^{\prime}\right)=\int_{\partial \Omega} G_{0}\left(E, \boldsymbol{x}\left(s^{\prime \prime}\right), \boldsymbol{y}(s)\right) \mu\left(s, s^{\prime}\right) \mathrm{d} s
$$

and it is a standard result from potential theory that this equation is solvable [19]. We can determine $\mu\left(s, s^{\prime}\right)$ from the jump relations for a single-layer potential [19]

$\partial_{n_{x}}^{ \pm} S L_{\mu}\left(\boldsymbol{x}(s), s^{\prime}\right)=\int_{\partial \Omega} \partial_{n_{x}} G_{0}\left(E, \boldsymbol{x}(s), \boldsymbol{y}\left(s^{\prime \prime}\right)\right) \mu\left(s^{\prime \prime}, s^{\prime}\right) \mathrm{d} s^{\prime \prime} \pm \frac{1}{2} \mu\left(s, s^{\prime}\right)$

where $\partial_{n_{x}}^{ \pm}$denotes interior (+) or exterior (-) limits of the normal derivative, i.e., for a function $\phi(\boldsymbol{x})$ they are defined as $\partial_{n_{x}}^{ \pm} \phi(\boldsymbol{x}(s)):=\lim _{\varepsilon \rightarrow 0}\left\langle\boldsymbol{n}(s), \nabla_{\boldsymbol{x}} \phi(\boldsymbol{x}(s) \mp \varepsilon \boldsymbol{n}(s))\right\rangle$ where $\boldsymbol{n}(s)$ denotes the outer normal unit vector to the boundary at $\boldsymbol{x}(s)$. From equation (19) and the jump relations (21) we then obtain

$$
g\left(k, s, s^{\prime}\right)=\partial_{n_{x}}^{+} f\left(\boldsymbol{x}(s), s^{\prime}\right)-\partial_{n_{x}}^{-} f\left(\boldsymbol{x}(s), s^{\prime}\right)=\mu\left(s, s^{\prime}\right) .
$$

Applying now $\partial_{n_{x}}^{+}$to equation (19) and using (21) and (22) leads to the desired integral equation on the boundary

$$
g\left(k, s, s^{\prime}\right)=g_{0}\left(k, s, s^{\prime}\right)-\int_{\partial \Omega} h_{1}\left(k, s, s^{\prime \prime}\right) g\left(k, s^{\prime \prime}, s^{\prime}\right) \mathrm{d} s^{\prime \prime}
$$

where $g\left(k, s, s^{\prime}\right)$ is given by (16) and

$$
\begin{aligned}
& g_{0}\left(k, s, s^{\prime}\right)=2 \partial_{n_{x}} \partial_{n_{y}} G_{0}\left(k^{2}, \boldsymbol{x}(s), \boldsymbol{y}\left(s^{\prime}\right)\right) \\
& h_{1}\left(k, s, s^{\prime}\right)=2 \partial_{n_{x}} G_{0}\left(k^{2}, \boldsymbol{x}(s), \boldsymbol{y}\left(s^{\prime}\right)\right) .
\end{aligned}
$$


Equation (23) is a Fredholm integral equation of second kind and can be solved by iteration. If we write $\boldsymbol{G}, \boldsymbol{G}_{0}, \boldsymbol{H}_{1}$ for the operators with integral kernels $g, g_{0}$ and $h_{1}$, respectively, the integral equation becomes

$$
G=G_{0}-H_{1} G
$$

which can be solved for $G$ as

$$
\boldsymbol{G}=\left(1+\boldsymbol{H}_{1}\right)^{-1} \boldsymbol{G}_{0}=\sum_{n \in \mathrm{N}_{0}}(-1)^{n} \boldsymbol{H}_{1}^{n} \boldsymbol{G}_{0}
$$

and the series converges if $\boldsymbol{H}_{1}$ is small enough. Going back to the kernels of the operators this gives the expansion

$$
g\left(k, s, s^{\prime}\right)=g_{0}\left(k, s, s^{\prime}\right)+\sum_{n=1}^{\infty}(-1)^{n} \int_{\partial \Omega} h_{n}\left(k, s, s^{\prime \prime}\right) g_{0}\left(k, s^{\prime \prime}, s^{\prime}\right) \mathrm{d} s^{\prime \prime}
$$

where for $n \geqslant 2$ we have

$h_{n}\left(k, s, s^{\prime \prime}\right)=\int_{\partial \Omega} \int_{\partial \Omega} \cdots \int_{\partial \Omega} h_{1}\left(k, s, s_{1}\right) h_{1}\left(k, s_{1}, s_{2}\right) \cdots h_{1}\left(k, s_{n-1}, s^{\prime \prime}\right) \mathrm{d} s_{1} \mathrm{~d} s_{2} \cdots \mathrm{d} s_{n-1}$.

Let us now discuss the convergence of the series (26). From the integral representation (64) for $h_{1}\left(k, s, s^{\prime}\right)$ (derived in appendix A) we see that for $s \neq s^{\prime}$ a positive imaginary part $\operatorname{Im} k$ gives an exponential damping factor, therefore we expect that there exists a $\gamma>0$ such that for $\operatorname{Im} k \geqslant \gamma$ the operator norm of $\boldsymbol{H}_{1}$ satisfies

$$
\left\|\boldsymbol{H}_{1}\right\|<1 \text {. }
$$

Then, for $\operatorname{Im} k \geqslant \gamma$, the series (26) would converge to a bounded operator. On the other hand, the boundary integral method tells us that the operator $\boldsymbol{H}_{1}$ has an eigenvalue -1 if $k^{2}$ is an eigenvalue of the Helmholtz equation (1). At these values the series (26) clearly diverges, as it should, since $g$ has a pole there.

It is often useful to consider spectral functions different from $g\left(k, s, s^{\prime}\right)$, and one way of introducing them such that the series (27) can still be applied is based on the formula

$$
\lim _{\gamma \rightarrow 0^{+}}-\frac{1}{\pi} \operatorname{Im} \frac{2(x+\mathrm{i} \gamma)}{(x+\mathrm{i} \gamma)^{2}-k^{2}}=\delta(x-k)+\delta(x+k) .
$$

Let $\rho(k)$ and $a(k)$ be functions which are holomorphic in the strip $-\varepsilon<\operatorname{Im} k<\gamma+\varepsilon$ for some $\varepsilon>0$, real valued for real arguments and $a(k)$ even. Furthermore, assume that their product $a(k) \rho(k)$ decays faster than $1 / k^{2}$ for $\operatorname{Re} k \rightarrow \pm \infty$. Then we define

$$
g^{\rho}\left(k, s, s^{\prime}\right):=-\frac{1}{\pi} \operatorname{Im} \int_{-\infty+\mathrm{i} \gamma}^{\infty+\mathrm{i} \gamma} 2 z \rho(k-z) a(z) g\left(z, s, s^{\prime}\right) \mathrm{d} z .
$$

Under the assumption (29) for $\operatorname{Im} k \geqslant \gamma$ we can insert expansion (27) and obtain a series which converges to the kernel of a bounded operator. On the other hand, since $g^{\rho}\left(k, s, s^{\prime}\right)$ is holomorphic in $\{k \in \mathrm{C} \mid \operatorname{Im} k>0\}$, integral (31) does not depend on $\gamma$. By taking the limit $\gamma \rightarrow 0^{+}$and using (30) and the definition (16) we get the representation

$$
g^{\rho}\left(k, s, s^{\prime}\right)=\sum_{n \in \mathrm{N}}\left[\rho\left(k-k_{n}\right)+\rho\left(k+k_{n}\right)\right] a\left(k_{n}\right) u_{n}(s) u_{n}^{*}\left(s^{\prime}\right) .
$$

So the role of the function $\rho$ is to select a spectral window in the summation, whereas $a(k)$ acts as a weight function. 
Now $\rho(k)$ and $a(k)$ can be adapted to the particular question one is interested in. In the next section, we want to study the mean behaviour of the boundary functions, and therefore will choose

$$
\rho(k)=\frac{1}{2 \pi} \int_{\mathrm{R}} \hat{\rho}(t) \mathrm{e}^{\mathrm{i} t k} \mathrm{~d} t
$$

where $\hat{\rho}$ is even and has compact support in a sufficiently small neighbourhood of 0 . This choice obviously fulfils the requirements needed. For the weight function $a(k)$ we will choose

$$
a(k)=\frac{1}{k^{2}+\alpha^{2}}
$$

with $\alpha>\gamma$. This also fulfils the requirements and satisfies $a(k)=1 / k^{2}+O\left(1 / k^{4}\right)$ for $k \rightarrow \infty$, which gives the correct normalization factor for the $u_{n}$ in view of (10).

\section{Mean behaviour of boundary functions}

In this section, we want to study the mean behaviour of the boundary functions $u_{n}$ for large energies $k_{n}^{2}$. We will do this by choosing a suitable test function $\rho$ in (31), namely we will assume that there is an $\varepsilon>0$ such that

$$
\operatorname{supp} \hat{\rho} \subset[-\varepsilon, \varepsilon] \quad \text { and } \quad \hat{\rho}(t)=1 \quad \text { for } t \in[-\varepsilon / 2, \varepsilon / 2]
$$

and furthermore that $\varepsilon$ is smaller than the shortest periodic orbit of the classical billiard flow. From (27), (28) and (31) we obtain the expansion

$$
g^{\rho}\left(k, s, s^{\prime}\right)=\sum_{n \in \mathrm{N}_{0}} g_{n}^{\rho}\left(k, s, s^{\prime}\right)
$$

with

$g_{n}^{\rho}\left(k, s, s^{\prime}\right):=\frac{(-1)^{n+1}}{\pi} \operatorname{Im} \int_{-\infty+\mathrm{i} \gamma}^{\infty+\mathrm{i} \gamma} 2 z \rho(k-z) a(z) \int_{\partial \Omega} h_{n}\left(z, s, s^{\prime \prime}\right) g_{0}\left(z, s^{\prime \prime}, s^{\prime}\right) \mathrm{d} s^{\prime \prime} \mathrm{d} z$

for $n \geqslant 1$, and for $n=0$

$$
g_{0}^{\rho}\left(k, s, s^{\prime}\right):=-\frac{1}{\pi} \operatorname{Im} \int_{-\infty+\mathrm{i} \gamma}^{\infty+\mathrm{i} \gamma} 2 z \rho(k-z) a(z) g_{0}\left(z, s, s^{\prime}\right) \mathrm{d} z .
$$

If $\rho$ is furthermore positive, the sum (32) can be interpreted as defining a mean value of boundary functions weighted with the factor $a\left(k_{n}\right)$, where the mean is taken over a spectral window around $k$ defined by $\rho$. In the following, we will assume that the billiard boundary is smooth, or can be obtained as the desymmetrization of a smooth billiard. We will show in appendix $\mathrm{C}$ that under the conditions (35) and with $a(k) \sim 1 / k^{2}$ for $k \rightarrow \infty$

$$
g_{n}^{\rho}(k, s, s)=O\left(k^{1-n}\right)
$$

and therefore the sum (36) provides an asymptotic expansion for large $k$ of the mean boundary functions.

The explicit computation of the first terms in the expansion (36) is given in appendix B. Here we choose $a$ to be of the form (34). For $s=s^{\prime}$ the first term is given by

$$
g_{0}^{\rho}(k, s, s)=\frac{k}{2 \pi}+O\left(k^{-\infty}\right)
$$

and for $s \sim s^{\prime}$ one has

$$
g_{0}^{\rho}\left(k, s, s^{\prime}\right)=\frac{k}{2 \pi}\left[\frac{2}{k\left|s-s^{\prime}\right|} J_{1}\left(k\left|s-s^{\prime}\right|\right)+O\left(s-s^{\prime}\right)\right]+O\left(k^{-\infty}\right) .
$$


The second term is for $s \sim s^{\prime}$ given by

$$
g_{1}^{\rho}\left(k, s, s^{\prime}\right)=-\frac{\kappa(s)}{2 \pi} \cos \left(2 k\left|s-s^{\prime}\right|\right)+O(1 / k)
$$

where $\kappa(s)$ denotes the curvature of the boundary at $s$.

Integrating $g^{\rho}\left(k, s, s^{\prime}\right)$ one obtains

$$
(N * \rho)\left(k, s, s^{\prime}\right)=\int_{0}^{k} g^{\rho}\left(k^{\prime}, s, s^{\prime}\right) \mathrm{d} k^{\prime}
$$

where $*$ denotes convolution and $N\left(k, s, s^{\prime}\right)$ is defined as

$$
N\left(k, s, s^{\prime}\right):=\sum_{k_{n} \leqslant k} \frac{u_{n}(s) u_{n}^{*}\left(s^{\prime}\right)}{k_{n}^{2}} .
$$

It is well known from the theory of spectral asymptotics that

$$
N\left(k, s, s^{\prime}\right)=(N * \rho)\left(k, s, s^{\prime}\right)(1+O(1 / k))
$$

see [18], and therefore from an asymptotic expansion of (43) we immediately obtain the leading semiclassical behaviour of (44).

Inserting (36) and using (40), (42) and (39) the asymptotic behaviour of (43) becomes

$$
(N * \rho)(k, s, s)=\frac{1}{4 \pi} k^{2}-\frac{\kappa(s)}{2 \pi} k+O(\ln k)
$$

which is the main result of this section.

Thus the leading term of (45) reads

$$
N(k, s, s)=\frac{1}{4 \pi} k^{2}+O(k)
$$

Assuming in addition that the set of $p \in[-1,1]$, such that $(p, s)$ belongs to a periodic orbit of the billiard map, has measure zero, we expect that the two-term asymptotics (46) holds for $N(k, s, s)$ as well, but with an error term $o(k)$. However, to prove this requires to adapt the more sophisticated methods from [18] or [7], where similar statements are proved for $N(k)$. Note that, as discussed in the introduction, the relation between $N(k)$ and $N(k, s, s)$ is given by

$$
N(k)=\frac{1}{2} \int_{\partial \Omega}\langle\boldsymbol{n}(s), \boldsymbol{x}(s)\rangle N(k, s, s) \mathrm{d} s
$$

and therefore we recover (6) from (46) using (10)-(12), up to the error term.

Often one studies billiards $\Omega$ with discrete symmetries and restricts the study to the corresponding symmetry subclasses of the eigenfunctions. For example, for a system which is symmetric with respect to reflection at the $x_{1}$-axis the eigenfunctions can be classified as either odd, fulfilling $\psi\left(x_{1}, x_{2}\right)=-\psi\left(x_{1},-x_{2}\right)$, or even, where $\psi\left(x_{1}, x_{2}\right)=\psi\left(x_{1},-x_{2}\right)$. Consequently, eigenfunctions with odd symmetry satisfy Dirichlet boundary conditions on the symmetry axis and even eigenfunctions obey Neumann boundary conditions on the symmetry axis. Of course, such symmetries of $\Omega$ induce symmetries of $\partial \Omega$. We restrict ourselves to the case of a reflection symmetry at a point $s_{0} \in \partial \Omega$. In this case the boundary Green function (13) has to be modified to

$$
g^{ \pm}\left(k, s, s^{\prime}\right)=g\left(k, s_{0}+s, s_{0}+s^{\prime}\right) \pm g\left(k, s_{0}+s, s_{0}-s^{\prime}\right)
$$

for $s, s^{\prime}$ near $s_{0}$ with even (+) or odd (-) symmetry. Using this and (41) we obtain for $s$ close to $s_{0}$

$$
g_{0}^{\rho \pm}(k, s, s)=\frac{k}{2 \pi}\left[1 \pm \frac{1}{k\left|s-s_{0}\right|} J_{1}\left(2 k\left|s-s_{0}\right|\right)\right](1+O(1 / k))
$$


and

$$
g_{1}^{\rho \pm}(k, s, s)=-\frac{\kappa(s)}{2 \pi}\left[1 \pm \cos \left(2 k\left|s-s_{0}\right|\right)\right](1+O(1 / k)) .
$$

Therefore, we get

$\left(N^{ \pm} * \rho\right)(k, s, s)=\frac{k^{2}}{4 \pi}\left[1 \pm \frac{1-J_{0}\left(2 k\left|s-s_{0}\right|\right)}{\left|s-s_{0}\right|^{2} k^{2}}\right]-k \frac{\kappa(s)}{2 \pi}\left[1 \pm \frac{\sin \left(2 k\left|s-s_{0}\right|\right)}{2 k\left|s-s_{0}\right|}\right]+O(\ln k)$

for $s$ close to a fixed point $s_{0}$ of the symmetry.

Our results show that the mean behaviour of the normalized boundary functions is very similar to the mean behaviour of eigenfunctions. The crucial difference between the two sequences of functions $\left\{\psi_{n}\right\}_{n \in \mathrm{N}},\left\{u_{n}\right\}_{n \in \mathrm{N}}$ is that the eigenfunctions live on a two-dimensional space whereas the boundary functions live on a one-dimensional space. Since both $u_{n}$ and $\psi_{n}$ oscillate roughly with the same de Broglie wavelength $2 \pi / k_{n}$, this leads to an overcompleteness of the set $\left\{u_{n}\right\}_{n \in \mathrm{N}}$. This statement can be made more explicit by observing that (41) implies

$$
g^{\rho}\left(k, s, s^{\prime}\right)=\frac{2}{\pi} \delta\left(s-s^{\prime}\right)+O(1 / k) .
$$

More precisely, this means that for every $\varphi \in C^{\infty}(\partial \Omega)$

$$
\varphi(s)=\sum_{n \in \mathrm{N}} \rho\left(k-k_{n}\right) \varphi_{n} u_{n}(s)+O(1 / k)
$$

holds with coefficients

$$
\varphi_{n}:=\frac{\pi}{2 k_{n}^{2}} \int_{\partial \Omega} u_{n}^{*}\left(s^{\prime}\right) \varphi\left(s^{\prime}\right) \mathrm{d} s^{\prime} .
$$

This follows from (72) by the method of stationary phase (see appendix D). Since $\rho$ is a rapidly decreasing function, this means that the boundary functions with spectral parameter $k_{n}$ in an interval of fixed width around $k$ form a complete set in the limit $k \rightarrow \infty$. The number of these states grows like $k$, in contrast to the number of all states up to energy $k^{2}$, which, according to the Weyl formula, grows like $k^{2}$. Therefore this result gives a quantitative measure of the overcompleteness of the set $\left\{u_{n}\right\}_{n \in \mathrm{N}}$.

\section{Numerical results for integrable, mixed and chaotic systems}

In this section we would like to test how well the asymptotic expansion (46) describes the mean behaviour of the boundary function at finite energies. To illustrate the energy dependence we plot in figure $2 N(k, s, s)$ for $s=3.2$ for the desymmetrized limaçon billiard with $\varepsilon=0.3$ (see the introduction). The dashed line beneath the staircase function (full curve) is the asymptotic result (46). As for the spectral staircase function excellent agreement, even down to the ground state, is observed. Note that for such a good agreement it is crucial to include the curvature term. This is illustrated by the dotted line in figure 2 which is a plot of the leading term $k^{2} /(4 \pi)$.

Now we turn to the $s$-dependence of $N(k, s, s)$, where for better comparison we have divided by $k^{2}$

$$
\sigma(k, s):=\frac{1}{k^{2}} \sum_{k_{n} \leqslant k} \frac{\left|u_{n}(s)\right|^{2}}{k_{n}^{2}} \equiv \frac{1}{k^{2}} N(k, s, s) .
$$

First, we consider the stadium billiard which is given by two semi-circles joined by two parallel straight lines. The stadium billiard is proved to be strongly chaotic, i.e. it is ergodic, mixing and a $K$-system $[20,21]$. The height of the desymmetrized billiard is chosen to be 1 , and $a$ 


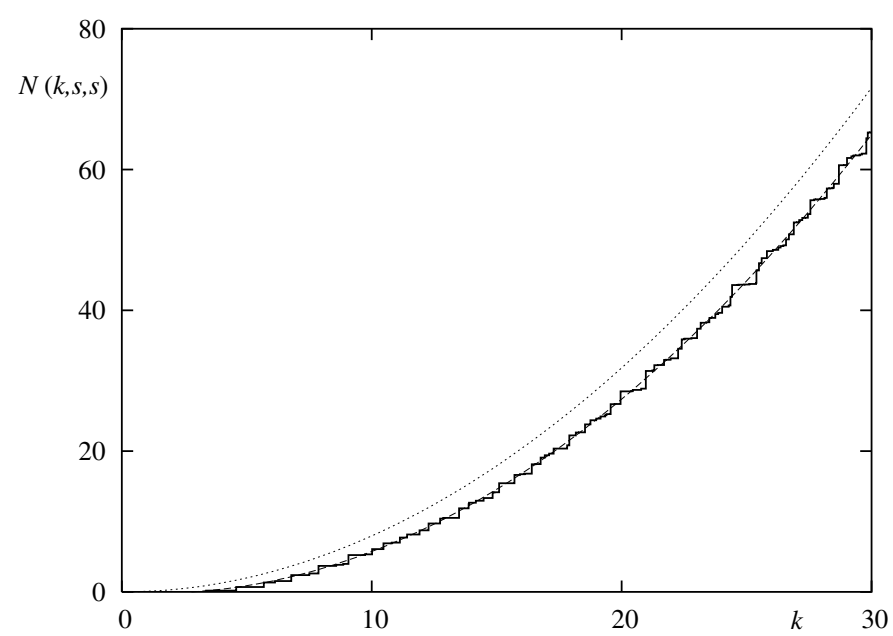

Figure 2. Plot of $N(k, s, s)$ at $s=3.2$ for the desymmetrized limaçon billiard with $\varepsilon=0.3$. The dashed curve is the asymptotic result (46) and the dotted curve is just the leading term (47).

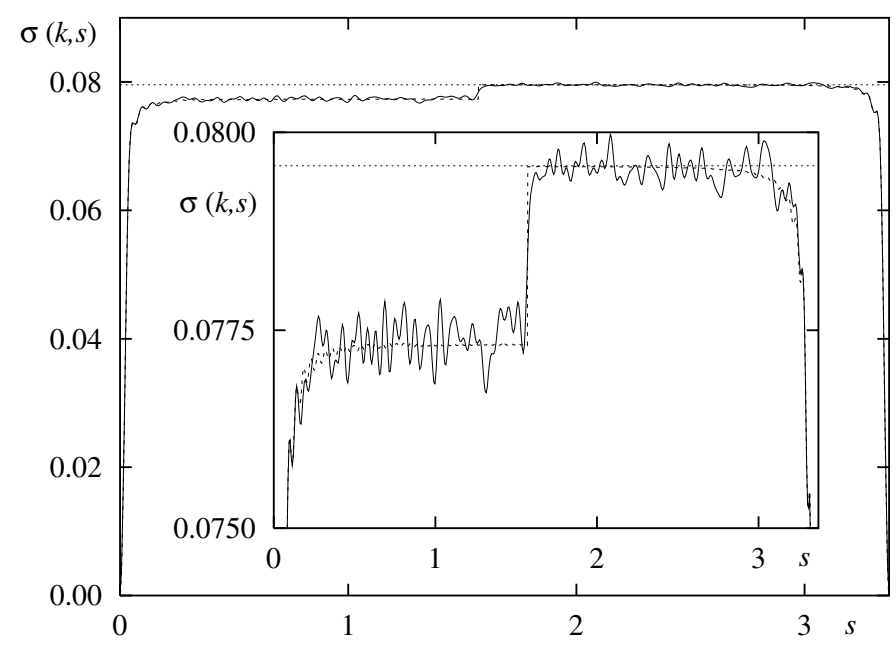

Figure 3. Plot of the spectral average $\sigma(k, s)$ for the fully desymmetrized stadium billiard with $a=1.8$ and Dirichlet boundary conditions everywhere. The energy $k^{2}$ is chosen such that the first 1000 boundary functions are taken into account. The horizontal dotted line is the leading term, $1 /(4 \pi)$, and the dashed line corresponds to the asymptotic formula $\bar{\sigma}^{-}(k, s)$, equation (57). The inset shows a magnification.

denotes the length of the upper horizontal line, for which we have $a=1.8$ in the following. The boundary is parametrized starting with $s=0$ at the corner of the quarter circle, ranging to $s=\pi / 2$ at the place where straight line and quarter circle join tangentially until $s=\pi / 2+a$ at the next corner. Figure 3 shows $\sigma(k, s)$ using the first 1000 boundary functions. The asymptotic result (see section 3 ) reads (for even and odd symmetry)

$\bar{\sigma}^{ \pm}(k, s):=\frac{1}{4 \pi}\left[1 \pm \sum_{\text {corners } i} \frac{1-J_{0}\left(2 k\left|s-s_{i}\right|\right)}{\left|s-s_{i}\right|^{2} k^{2}}\right]-\frac{\kappa(s)}{2 \pi k}\left[1 \pm \sum_{\text {corners } i} \frac{\sin \left(2 k\left|s-s_{i}\right|\right)}{2 k\left|s-s_{i}\right|}\right]$

where the contributions near the corners are taken into account. 


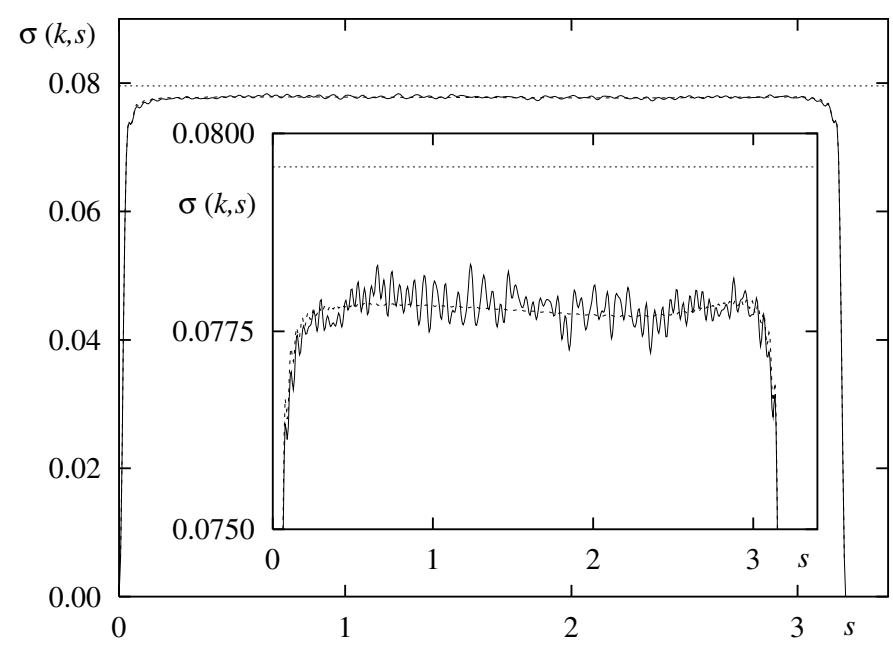

Figure 4. Same as in the previous figure but for the desymmetrized limaçon billiard with $\varepsilon=0.3$. For this parameter the classical phase space is mixed.

At $s=\pi / 2$ the mean behaviour shows a jump caused by the discontinuity in the curvature of the boundary (transition from the circular part to the straight line). Although our derivation is not valid at this point we observe an excellent agreement of $\sigma(k, s)$ with $\bar{\sigma}^{-}(k, s)$. Moreover, the behaviour near the two corners is clearly visible and very well described by $\bar{\sigma}^{-}(k, s)$.

The next example, shown in figure 4 for the limaçon billiard with $\varepsilon=0.3$, illustrates that the mean behaviour is independent of the classical dynamical properties as for this parameter the considered billiard is a mixed system (i.e. regular and irregular regions in phase space coexist). The asymptotic formula also works well in cases where the curvature changes more strongly as for example for the limaçon billiard with $\varepsilon=0.7$ (see figure 5). In this case the classical dynamics appears to be ergodic (though it is not the case since there exist some very small islands of stability [22]).

Finally, we would like to consider the circle billiard as an example of an integrable system. The boundary functions of a circle billiard with radius $R$ and the corresponding eigenvalues are given by

$$
u_{l m}(s)=-\frac{j_{l m}}{\sqrt{\pi} R^{2}} \mathrm{e}^{\mathrm{i} l \frac{s}{R}} \quad \text { and } \quad k_{l m}^{2}=\left(\frac{j_{l m}}{R}\right)^{2}
$$

respectively, where $j_{l m}$ denotes the $m$ th positive zero of the $J_{l}$-Bessel function. Therefore,

$$
\sum_{k_{l m} \leqslant k} \frac{\left|u_{l m}(s)\right|^{2}}{k_{l m}^{2}}=\frac{1}{\mathcal{A}} N(k) .
$$

Turning to the desymmetrized circle billiard no such simple expression exists. Figure 6 shows a plot of $\sigma(k, s)$ for the desymmetrized circle billiard with Neumann boundary condition on the symmetry axis. Again, we find very good agreement with the corresponding asymptotic result $\bar{\sigma}^{+}(k, s)$.

\section{Summary}

We have studied the semiclassical behaviour of the normal derivative of eigenfunctions of Euclidean billiards. These boundary functions form a reduced representation of the quantum 


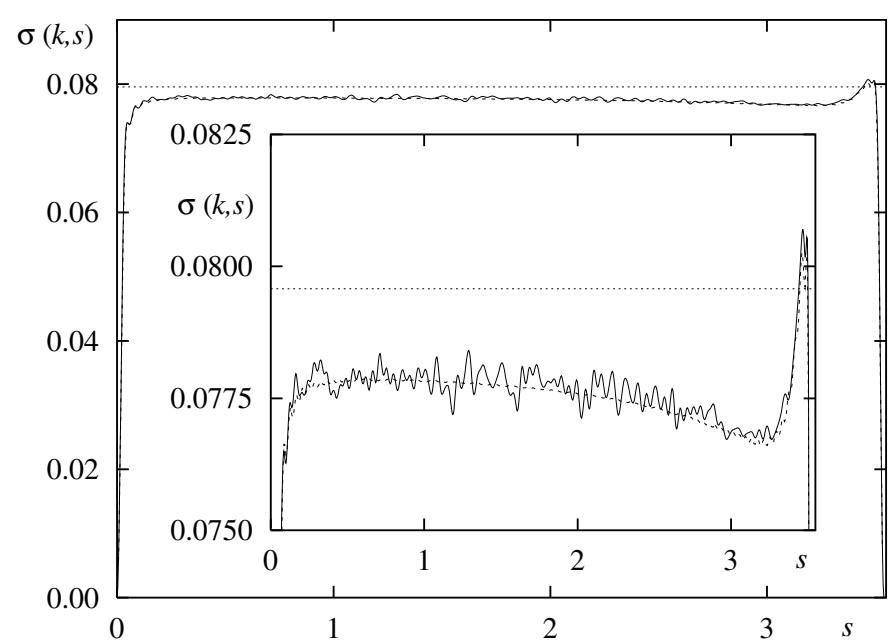

Figure 5. Same as in the previous figure but for the desymmetrized limaçon billiard with $\varepsilon=0.7$. Here the influence of the curvature contribution is clearly visible.

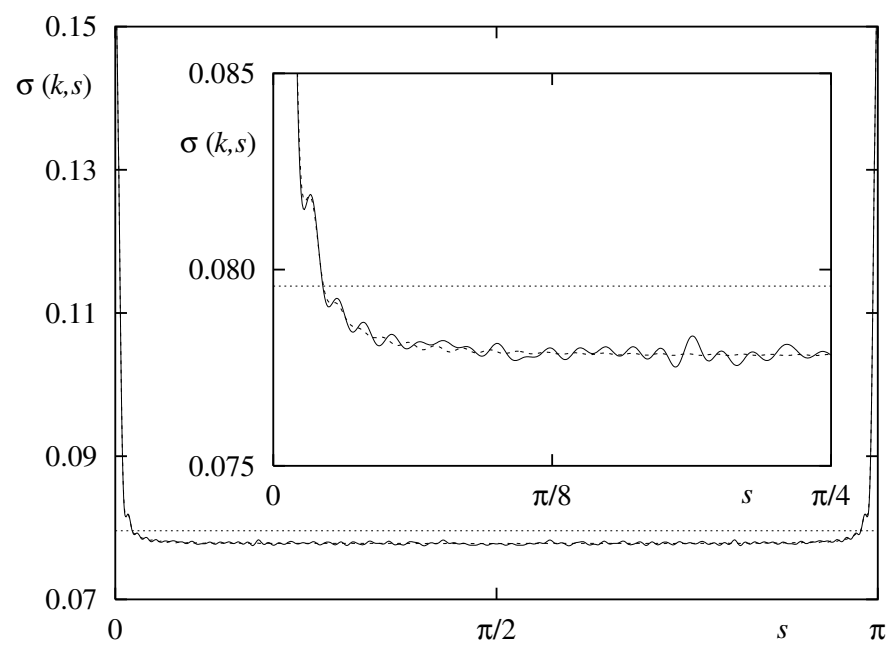

Figure 6. Plot of $\sigma(k, s)$ for the desymmetrized circle billiard with radius one and Neumann boundary condition compared with the corresponding asymptotic result $\bar{\sigma}^{+}(k, s)$ shown as dashed line.

system, analogous to the Poincare section of the classical billiard. Thus, they are of special interest in order to understand the properties of the eigenfunctions of billiards and their relation to the classical system, as investigated in the field of quantum chaos.

We have introduced an analogue of the standard energy Green function for the boundary functions, and one of our main results is the derivation of an integral equation for this boundary Green function. The resulting integral equation is the principal tool for the semiclassical analysis and leads to an expansion of the boundary Green function which is the analogue of the classical multiple reflection expansion. The resulting expansion could now be used to derive a semiclassical representation of the boundary Green function in terms of orbits of the 
classical billiard map, but here we were only interested in the so-called length-zero contribution which determines the mean behaviour of the normal derivatives for large energies.

For the mean behaviour of the sequence of the boundary functions we have derived a two-term asymptotics for large energies, where the first term is universal, i.e. completely independent of the given billiard, and the second term is proportional to the curvature of the boundary. These theoretical results fit very well with the numerical computations for different Euclidean billiards.

Furthermore, we have obtained a completeness relation for the boundary functions with momenta $k_{n}$ near $k$ for large $k$, which shows that the whole set of normal derivatives is highly over-complete, since a fraction of order $k$ is asymptotically sufficient to span the reduced state space over the boundary.

\section{Acknowledgments}

This research was supported in part by the DFG grants Ba 1973/1-1 and Ste 241/7-3 and the EC Research Training Network under contract no HPRN-CT-2000-00103.

\section{Appendix A. Integral representations}

For the computation of the two leading terms in the expansion (36), equations (40) and (42), we need suitable integral representations of $h_{1}\left(k, s, s^{\prime}\right)$ and $g_{0}\left(k, s, s^{\prime}\right)$. We start by deriving a representation of $h_{1}\left(k, s, s^{\prime}\right)$. Namely, from (17) and (25) we get

$$
h_{1}\left(k, s, s^{\prime}\right)=\frac{2 \mathrm{i}}{(2 \pi)^{2}} \int_{\mathrm{R}^{2}} \frac{\langle\boldsymbol{n}(s), \boldsymbol{\xi}\rangle}{E-|\boldsymbol{\xi}|^{2}} \mathrm{e}^{\mathrm{i}\left\langle\boldsymbol{\xi}, \boldsymbol{x}(s)-\boldsymbol{y}\left(s^{\prime}\right)\right\rangle} \mathrm{d}^{2} \xi
$$

where $E=k^{2}$. Inserting the relation

$$
\frac{1}{E-|\xi|^{2}}=-\mathrm{i} \varepsilon \int_{0}^{\infty} \mathrm{e}^{\mathrm{i} \varepsilon\left(E-|\xi|^{2}\right) t} \mathrm{~d} t
$$

with $\varepsilon=\operatorname{sgn} \operatorname{Im} E$ and for $|\operatorname{Im} E|>0$, allows us to solve the $\xi$-integral and we arrive at

$$
h_{1}\left(k, s, s^{\prime}\right)=-\frac{\mathrm{i} \varepsilon}{\pi} \int_{0}^{\infty} \frac{\left\langle\boldsymbol{n}(s), \delta \boldsymbol{x}\left(s, s^{\prime}\right)\right\rangle}{4 t^{2}} \mathrm{e}^{\mathrm{i} \varepsilon\left[\frac{\left|\delta \boldsymbol{x}\left(s, s^{\prime}\right)\right|^{2}}{4 t}+k^{2} t\right]} \mathrm{d} t
$$

where we have used the abbreviation $\delta \boldsymbol{x}\left(s, s^{\prime}\right)=\boldsymbol{x}(s)-\boldsymbol{y}\left(s^{\prime}\right)$. This expression has the disadvantage that the factor $\left\langle\boldsymbol{n}(s), \delta \boldsymbol{x}\left(s, s^{\prime}\right)\right\rangle$ tends to zero for $s \rightarrow s^{\prime}$, so we use

$$
-\frac{1}{\left|\delta \boldsymbol{x}\left(s, s^{\prime}\right)\right|^{2}} \frac{\mathrm{d}}{\mathrm{d} t} \mathrm{e}^{\mathrm{i} \varepsilon \frac{\left|\delta x\left(s, s^{\prime}\right)\right|^{2}}{4 t}}=\frac{\mathrm{i} \varepsilon}{4 t^{2}} \mathrm{e}^{\mathrm{i} \varepsilon \frac{\left|\delta x\left(s, s^{\prime}\right)\right|^{2}}{4 t}}
$$

and partial integration (the boundary term at $t=0$ vanishes in the weak sense as a function of $s$ ) to obtain

$$
h_{1}\left(k, s, s^{\prime}\right)=-\frac{\mathrm{i} \varepsilon k^{2}}{\pi} \frac{\left\langle\boldsymbol{n}(s), \delta \boldsymbol{x}\left(s, s^{\prime}\right)\right\rangle}{\left|\delta \boldsymbol{x}\left(s, s^{\prime}\right)\right|^{2}} \int_{0}^{\infty} \mathrm{e}^{\mathrm{i} \varepsilon\left[\frac{\left|\delta \boldsymbol{x}\left(s, s^{\prime}\right)\right|^{2}}{4 t}+k^{2} t\right]} \mathrm{d} t .
$$

Note that for $s \sim s^{\prime}$

$$
\frac{\left\langle\boldsymbol{n}(s), \delta \boldsymbol{x}\left(s, s^{\prime}\right)\right\rangle}{\left|\delta \boldsymbol{x}\left(s, s^{\prime}\right)\right|^{2}}=\frac{1}{2} \kappa(s)+O\left(s-s^{\prime}\right)
$$

where $\kappa(s)$ denotes the curvature of the boundary $\partial \Omega$ at $\boldsymbol{x}(s)$ (with the sign convention that it is positive for a circle). 
A similar representation can be derived for $g_{0}\left(k, s, s^{\prime}\right)$. Inserting now (61) in (24) allows us again to compute the $\xi$-integral and we obtain

$$
\begin{aligned}
g_{0}\left(k, s, s^{\prime}\right)= & -\frac{1}{2 \pi} \int_{0}^{\infty}\left[\frac{\left\langle\boldsymbol{n}(s), \delta \boldsymbol{x}\left(s, s^{\prime}\right)\right\rangle\left\langle\boldsymbol{n}\left(s^{\prime}\right), \delta \boldsymbol{x}\left(s, s^{\prime}\right)\right\rangle}{4 r^{3}}\right. \\
& \left.-\mathrm{i} \varepsilon \frac{\left\langle\boldsymbol{n}(s), \boldsymbol{n}\left(s^{\prime}\right)\right\rangle}{2 r^{2}}\right] \mathrm{e}^{\mathrm{i} \varepsilon\left[\frac{1}{4 r}\left(\delta \boldsymbol{x}\left(s, s^{\prime}\right)\right)^{2}+k^{2} r\right]} \mathrm{d} r .
\end{aligned}
$$

The integral is again well defined as an oscillatory integral, but it will be useful below to have a suitable regularization at hand when we want to change the order of integration. To this end, let $G_{0, \zeta}\left(k^{2}, \boldsymbol{x}, \boldsymbol{y}\right)$ be the integral kernel of the complex power of the free resolvent, $\left(-\Delta+k^{2}\right)^{-\zeta}, \zeta \in \mathrm{C}$, and let $g_{0, \zeta}\left(k, s, s^{\prime}\right):=2 \partial_{n_{x}} \partial_{n_{y}} G_{0, \zeta}\left(k^{2}, \boldsymbol{x}(s), \boldsymbol{y}\left(s^{\prime}\right)\right)$. Then a calculation analogous to the one leading to (66), using $\left(E-|\xi|^{2}\right)^{-\zeta}=\frac{\mathrm{e}^{-\mathrm{i} \frac{\pi}{2} \zeta} \xi^{\zeta}}{\Gamma(\zeta)} \int_{0}^{\infty} r^{\zeta-1} \mathrm{e}^{\mathrm{i} \varepsilon\left(E-|\xi|^{2}\right) r} \mathrm{~d} r$ instead of (61), shows that

$$
\begin{aligned}
g_{0, \zeta}\left(k, s, s^{\prime}\right)= & -\frac{\varepsilon^{\zeta+1} \mathrm{e}^{-\mathrm{i} \frac{\pi}{2}(\zeta-1)}}{2 \pi \Gamma(\zeta)} \int_{0}^{\infty}\left[\frac{\left\langle\boldsymbol{n}(s), \delta \boldsymbol{x}\left(s, s^{\prime}\right)\right\rangle\left\langle\boldsymbol{n}\left(s^{\prime}\right), \delta \boldsymbol{x}\left(s, s^{\prime}\right)\right\rangle}{4 r^{3}}\right. \\
& \left.-\mathrm{i} \varepsilon \frac{\left\langle\boldsymbol{n}(s), \boldsymbol{n}\left(s^{\prime}\right)\right\rangle}{2 r^{2}}\right] r^{\zeta-1} \mathrm{e}^{\mathrm{i} \varepsilon\left[\frac{1}{4 r}\left(\delta \boldsymbol{x}\left(s, s^{\prime}\right)\right)^{2}+k^{2} r\right]} \mathrm{d} r
\end{aligned}
$$

and we have of course $g_{0,1}\left(k, s, s^{\prime}\right)=g_{0}\left(k, s, s^{\prime}\right)$.

\section{Appendix B. The computation of $g_{0}^{\rho}$ and $g_{1}^{\rho}$}

In this appendix, we will use the integral representations derived in appendix A to compute the first and second terms in the asymptotic expansion (36).

We start with the first term, (38). Since the integrand is holomorphic in the upper half-plane, we can take the limit $\gamma \rightarrow 0$, and using (24) we obtain

$$
g_{0}^{\rho}\left(k, s, s^{\prime}\right)=\frac{2}{(2 \pi)^{2}} \int_{\mathrm{R}^{2}}[\rho(k-|\boldsymbol{\xi}|)+\rho(k+|\boldsymbol{\xi}|)] a(|\boldsymbol{\xi}|)\langle\boldsymbol{n}(s), \boldsymbol{\xi}\rangle\left\langle\boldsymbol{n}\left(s^{\prime}\right), \boldsymbol{\xi}\right\rangle \mathrm{e}^{\mathrm{i}\left\langle\delta \boldsymbol{x}\left(s, s^{\prime}\right), \boldsymbol{\xi}\right\rangle} \mathrm{d}^{2} \xi .
$$

Since $\rho$ is rapidly decreasing, and $|\boldsymbol{\xi}|$ is positive, the term $\rho(k+|\boldsymbol{\xi}|)$ is smaller than any negative power of $k$ for large $k$ and can therefore be neglected. Using representation (33) and introducing polar coordinates in the $\xi$-integral leads to

$$
\begin{aligned}
g_{0}^{\rho}\left(k, s, s^{\prime}\right)= & \frac{2 k^{4}}{(2 \pi)^{3}} \int_{0}^{2 \pi} \int_{0}^{\infty} \int_{-\infty}^{\infty} \hat{\rho}(t) a(k r)\langle\boldsymbol{n}(s), \hat{e}(\varphi)\rangle\left\langle\boldsymbol{n}\left(s^{\prime}\right), \hat{e}(\varphi)\right\rangle \\
& \times \mathrm{e}^{\mathrm{i} k\left[t(1-r)+r\left\langle\delta \boldsymbol{x}\left(s, s^{\prime}\right), \hat{e}(\varphi)\right\rangle\right]} r^{3} \mathrm{~d} t \mathrm{~d} r \mathrm{~d} \varphi+O\left(k^{-\infty}\right)
\end{aligned}
$$

where $\hat{e}(\varphi)$ denotes the unit vector in direction $\varphi$. The $r, t$-integrals can now be evaluated and give

$$
\begin{aligned}
\frac{k}{2 \pi} \int_{-\infty}^{\infty} \int_{0}^{\infty} & \hat{\rho}(t) \mathrm{e}^{\mathrm{i} k\left[t(1-r)+r\left\langle\delta x\left(s, s^{\prime}\right), \hat{e}(\varphi)\right\rangle\right]} a(r k) r^{3} \mathrm{~d} t \mathrm{~d} r \\
& =\frac{k}{2 \pi} \int_{-\infty}^{\infty} \int_{-1}^{\infty} \hat{\rho}\left(t+\left\langle\delta \boldsymbol{x}\left(s, s^{\prime}\right), \hat{e}(\varphi)\right\rangle\right) \mathrm{e}^{-\mathrm{i} k t r} a((r+1) k)(r+1) \mathrm{d} r \mathrm{~d} t \mathrm{e}^{\mathrm{i} k\left\langle\delta \boldsymbol{x}\left(s, s^{\prime}\right), \hat{e}(\varphi)\right\rangle} \\
& =\left.\mathrm{e}^{\frac{\mathrm{i}}{k} \partial_{t} \partial_{r}} \hat{\rho}\left(t+\left\langle\delta \boldsymbol{x}\left(s, s^{\prime}\right), \hat{e}(\varphi)\right\rangle\right) a((r+1) k)(1+r)\right|_{r=t=0} \mathrm{e}^{\mathrm{i} k\left\langle\delta x\left(s, s^{\prime}\right), \hat{e}(\varphi)\right\rangle}+O\left(k^{-\infty}\right) \\
& =a(k) \mathrm{e}^{\mathrm{i} k\left\langle\delta \boldsymbol{x}\left(s, s^{\prime}\right), \hat{e}(\varphi)\right\rangle}+O\left(k^{-\infty}\right)
\end{aligned}
$$


for $\left|\left\langle\delta \boldsymbol{x}\left(s, s^{\prime}\right), \hat{\boldsymbol{e}}(\varphi)\right\rangle\right|<\varepsilon / 2$ by (35). In the step from the second to the third line we have used the general relation

$$
\frac{k}{2 \pi} \int_{-\infty}^{\infty} \int_{-\infty}^{\infty} \mathrm{e}^{-\mathrm{i} k r t} f(r, t) \mathrm{d} r \mathrm{~d} t=\left.\mathrm{e}^{-\frac{\mathrm{i}}{k} \partial_{r} \partial_{t}} f(r, t)\right|_{r=0, t=0}
$$

The error $O\left(k^{-\infty}\right)$ is due to the cut-off of the $r$-integral at $r=-1$. This finally leads to

$g_{0}^{\rho}\left(k, s, s^{\prime}\right)=\frac{a(k) k^{3}}{2 \pi^{2}} \int_{0}^{2 \pi}\langle\boldsymbol{n}(s), \hat{e}(\varphi)\rangle\left\langle\boldsymbol{n}\left(s^{\prime}\right), \hat{e}(\varphi)\right\rangle \mathrm{e}^{\mathrm{i} k\left\langle\delta \boldsymbol{x}\left(s, s^{\prime}\right), \hat{e}(\varphi)\right\rangle} \mathrm{d} \varphi+O\left(k^{-\infty}\right)$

for $s$ close to $s^{\prime}$. At $s=s^{\prime}$ we get

$$
g_{0}^{\rho}(k, s, s)=\frac{a(k) k^{3}}{2 \pi}+O\left(k^{-\infty}\right)
$$

and for $s \sim s^{\prime}$ a Taylor expansion of the exponent gives

$$
\begin{aligned}
g_{0}^{\rho}\left(k, s, s^{\prime}\right) & \approx \frac{a(k) k^{3}}{2 \pi^{2}} \int_{0}^{2 \pi} \cos ^{2} \phi \mathrm{e}^{\mathrm{i} k\left|s-s^{\prime}\right| \sin \phi} \mathrm{d} \phi \\
& =\frac{a(k) k^{3}}{2 \pi} \frac{2}{k\left|s-s^{\prime}\right|} J_{1}\left(k\left|s-s^{\prime}\right|\right) .
\end{aligned}
$$

For the determination of the second term, $g_{1}^{\rho}\left(k, s, s^{\prime}\right)$, it is useful to use the regularized expression (67) with $\zeta$ in a range where all integrals converge, and finally make an analytic continuation to $\zeta=1$. So we use representations (64) and (67) and insert them in (37). The $k$-dependence in the expressions for $g_{0, \zeta}$ and $h_{1}$ is simple and we can perform the resulting $z$-integral

$$
\frac{1}{\pi} \int_{-\infty+\mathrm{i} \gamma}^{\infty+\mathrm{i} \gamma} 2 z \rho(k-z) a(z) h_{1}\left(z, s, s^{\prime \prime}\right) g_{0, \zeta}\left(z, s^{\prime \prime}, s^{\prime}\right) \mathrm{d} z .
$$

Since the integrand is holomorphic in the upper half-plane, we can perform the limit $\gamma \rightarrow 0$. If we furthermore change variables $r \rightarrow r / k$ and $t \rightarrow t / k$ in (66) and (64), and interchange the order of integration, the $z$-integral boils down to

$$
\begin{gathered}
\frac{1}{\pi} \int_{-\infty}^{\infty} 2 z^{3} \rho(k-z) a(z) \mathrm{e}^{\mathrm{i} \frac{\mathrm{t}+r}{k} z^{2}} \mathrm{~d} z=\frac{1}{\pi} \mathrm{e}^{\mathrm{i} k(t+r)} \int_{-\infty}^{\infty} 2(k-z)^{3} a(k-z) \rho(z) \mathrm{e}^{\mathrm{i} \frac{t+r}{k} z^{2}} \mathrm{e}^{-\mathrm{i} 2(t+r) z} \mathrm{~d} z \\
=\frac{2 k^{3} a(k)}{\pi} \hat{\rho}(2(t+r)) \mathrm{e}^{\mathrm{i} k(t+r)}(1+O(1 / k)) .
\end{gathered}
$$

Here we need that $a$ satisfies a kind of symbol estimate. Collecting the remaining integrals leads to

$$
\begin{aligned}
g_{1, \zeta}^{\rho}\left(k, s, s^{\prime}\right)= & \operatorname{Im}\left[\frac{k^{4-\zeta} a(k)}{\pi^{3}} \int_{0}^{\infty} \int_{0}^{\infty} \int_{\partial \Omega} b\left(k, s, s^{\prime}, s^{\prime \prime}, r, \zeta\right) \hat{\rho}(2(t+r))(1+O(1 / k))\right. \\
& \left.\times \mathrm{e}^{\mathrm{i} k\left[\frac{1}{4 r}\left(\delta \boldsymbol{x}\left(s^{\prime \prime}, s^{\prime}\right)\right)^{2}+\frac{1}{4 t}\left(\delta x\left(s, s^{\prime \prime}\right)\right)^{2}+(r+t)\right]} \mathrm{d} s^{\prime \prime} \mathrm{d} r \mathrm{~d} t\right]
\end{aligned}
$$

with

$$
\begin{aligned}
b\left(k, s, s^{\prime}, s^{\prime \prime}, r, \zeta\right) & =\mathrm{e}^{-\mathrm{i} \frac{\pi}{2}(\zeta-1)} \frac{\left\langle\boldsymbol{n}(s), \delta \boldsymbol{x}\left(s, s^{\prime \prime}\right)\right\rangle}{\left|\delta \boldsymbol{x}\left(s, s^{\prime \prime}\right)\right|^{2}} \\
\times & {\left[\mathrm{i} k \frac{\left\langle\boldsymbol{n}\left(s^{\prime \prime}\right), \delta \boldsymbol{x}\left(s^{\prime \prime}, s^{\prime}\right)\right\rangle\left\langle\boldsymbol{n}\left(s^{\prime}\right), \delta \boldsymbol{x}\left(s^{\prime \prime}, s^{\prime}\right)\right\rangle}{4 r^{3}}+\frac{\left\langle\boldsymbol{n}\left(s^{\prime \prime}\right), \boldsymbol{n}\left(s^{\prime}\right)\right\rangle}{2 r^{2}}\right] r^{\zeta-1} . }
\end{aligned}
$$


This looks quite complicated and to understand the properties of this expression better let us discuss the stationary points of the phase function $\frac{1}{4 r}\left(\delta \boldsymbol{x}\left(s^{\prime \prime}, s^{\prime}\right)\right)^{2}+\frac{1}{4 t}\left(\delta \boldsymbol{x}\left(s, s^{\prime \prime}\right)\right)^{2}+(r+t)$ with respect to $s^{\prime \prime}, t, r$. The derivatives with respect to $t$ and $r$ give the conditions

$$
-\frac{1}{4 t^{2}}\left(\delta \boldsymbol{x}\left(s, s^{\prime \prime}\right)\right)^{2}+1=0 \quad-\frac{1}{4 r^{2}}\left(\delta \boldsymbol{x}\left(s^{\prime \prime}, s^{\prime}\right)\right)^{2}+1=0
$$

respectively, and hence $2 t=\left|\delta \boldsymbol{x}\left(s, s^{\prime \prime}\right)\right|$ and $2 r=\left|\delta \boldsymbol{x}\left(s^{\prime \prime}, s^{\prime}\right)\right|$. The $s^{\prime \prime}$-derivative leads to

$$
\frac{1}{2 r}\left\langle\boldsymbol{t}\left(s^{\prime \prime}\right), \delta \boldsymbol{x}\left(s^{\prime \prime}, s^{\prime}\right)\right\rangle-\frac{1}{2 t}\left\langle\boldsymbol{t}\left(s^{\prime \prime}\right), \delta \boldsymbol{x}\left(s, s^{\prime \prime}\right)\right\rangle=0
$$

which yields together with the previous conditions on $t$ and $r$ for $s^{\prime \prime}$

$$
\left\langle\boldsymbol{t}\left(s^{\prime \prime}\right), \delta \widehat{\boldsymbol{x}\left(s^{\prime \prime}, s^{\prime}\right)}\right\rangle=\left\langle\boldsymbol{t}\left(s^{\prime \prime}\right), \delta \widehat{\boldsymbol{x}\left(s, s^{\prime \prime}\right)}\right\rangle .
$$

But this is just the condition that there exists a trajectory in the billiard $\Omega$ starting at the point $s$ at the boundary, which is then elastically reflected at $s^{\prime \prime}$ and ending in $s^{\prime}$. This is, of course, what one expects by analogy with similar expressions, namely that $g^{\rho}\left(k, s, s^{\prime}\right)$ is semiclassically given by a sum over all classical orbits from $s$ to $s^{\prime}$, each contributing an amplitude depending on the stability of the orbit and an oscillating factor with frequency proportional to the length of the orbit. The $n$th term in the expansion of $g^{\rho}\left(k, s, s^{\prime}\right)$ contains exactly the orbits with $n$ reflections on the boundary. For the determination of the contribution of these orbits in leading order one can simplify the formulae for $g_{0}$ and $h_{1}$ considerably by using their asymptotic expansions for large arguments which can easily be derived by the method of stationary phase from the integral representations in appendix A. But if $s$ is close to $s^{\prime}$, there is one very short orbit with $s^{\prime \prime}$ between $s$ and $s^{\prime}$ whose length tends to zero for $s \rightarrow s^{\prime}$. The contribution of this orbit determines the mean behaviour of $g^{\rho}(k, s, s)$ and therefore it is commonly called the length-zero contribution. For the computation of this contribution the above-mentioned asymptotic formulae for $g_{0}$ and $h_{1}$ cannot be used because they are not valid in these regions, hence we must work with the full representation as in (77).

The $s^{\prime \prime}$-integral can be solved by the method of stationary phase, and since $\hat{\rho}$ is supported in a small neighbourhood of 0 there is only one stationary point for $s$ close to $s^{\prime}$, and we find that $g_{1, \zeta}^{\rho}\left(k, s, s^{\prime}\right)$ is for $s \sim s^{\prime}$ equal to the imaginary part of

$$
\begin{aligned}
\frac{a(k) \kappa(s) k^{7 / 2-\zeta}}{2 \pi^{5 / 2}} & \Gamma(\zeta) \\
& \quad \times \mathrm{e}^{-\mathrm{i} k\left(\frac{\zeta\left(\frac{\zeta}{2}-\frac{3}{4}\right)^{2}}{4(t+r)}+(t+r)\right]} \hat{\rho}(2(t+r)) \mathrm{d} r \mathrm{~d} t(1+O(1 / k))
\end{aligned}
$$

Introducing now the coordinates $v=t+r$ and $w=t-r$ gives

$$
\begin{aligned}
& \int_{0}^{\infty} \int_{0}^{\infty} \frac{1}{r^{5 / 2-\zeta}}\left(\frac{t}{t+r}\right)^{1 / 2} \mathrm{e}^{\mathrm{i} k\left[\frac{\left(s-s^{\prime}\right)^{2}}{4(t+r)}+(t+r)\right]} \hat{\rho}(2(t+r)) \mathrm{d} r \mathrm{~d} t \\
&= 2^{1-\zeta} \int_{0}^{\infty} v^{-1 / 2} \int_{-v}^{v} \frac{(v+w)^{1 / 2}}{(v-w)^{5 / 2-\zeta}} \mathrm{d} w \mathrm{e}^{\mathrm{i} k\left[\frac{\left(s-s^{\prime}\right)^{2}}{4 v}+v\right]} \hat{\rho}(2 v) \mathrm{d} v \\
&= B\left(\frac{3}{2},-\frac{3}{2}+\zeta\right) \int_{0}^{\infty} v^{-3 / 2+\zeta} \mathrm{e}^{\mathrm{i} k\left[\frac{\left(s-s^{\prime}\right)^{2}}{4 v}+v\right]} \hat{\rho}(2 v) \mathrm{d} v \\
&= B\left(\frac{3}{2},-\frac{3}{2}+\zeta\right) \mathrm{i} \pi \hat{\rho}(0)\left(\frac{\left|s-s^{\prime}\right|}{2}\right)^{-\frac{1}{2}+\zeta} \\
& \times \mathrm{e}^{-\mathrm{i} \pi\left(\frac{\zeta}{2}-\frac{1}{4}\right)} H_{\frac{1}{2}-\zeta}^{(1)}\left(k\left|s-s^{\prime}\right|\right)[1+O(1 / k)]
\end{aligned}
$$


where $B(u, v)$ denotes the beta function and $H_{v}^{(1)}(x)$ the Hankel function of the first kind. After collecting all terms we can perform the analytic continuation to $\zeta=1$ and finally obtain

$$
g_{1}\left(k, s, s^{\prime}\right)=-\hat{\rho}(0) a(k) k^{2} \frac{\kappa(s)}{2 \pi} \cos \left(k\left|s-s^{\prime}\right|\right)[1+O(1 / k)]
$$

for $s \sim s^{\prime}$.

\section{Appendix C. Estimating $g_{n}^{\rho}$}

In this appendix we derive the estimate (39) on $g_{n}^{\rho}(k, s, s)$. For $g_{n}^{\rho}(k, s, s)$ one obtains a similar expression as (77),

$$
\begin{aligned}
g_{n}^{\rho}(k, s, s)=\operatorname{Im} & {\left[k^{n+1} \int_{0}^{\infty} \int_{\mathrm{R}_{+}^{n}} \int_{\partial \Omega^{n}} b\left(s, s^{\prime}, r\right) a(k) \hat{\rho}\left(2 r+2 \sum_{i=1}^{n} t_{i}\right)\right.} \\
& \left.\times \mathrm{e}^{\mathrm{i} k\left[\frac{1}{4 r}\left(\delta x\left(s^{\prime \prime}, s^{\prime}\right)\right)^{2}+r+\sum_{i=1}^{n+1} \frac{1}{4 t_{i}}\left(\delta x\left(s_{i-1}, s_{i}^{\prime}\right)\right)^{2}+\sum_{i=1}^{n} t_{i}\right]} \mathrm{d}^{n} s^{\prime} \mathrm{d}^{n} t \mathrm{~d} r\right]
\end{aligned}
$$

with

$$
\begin{aligned}
b\left(s, s^{\prime}, r\right)= & \prod_{i=1}^{n+1} \frac{\left\langle\boldsymbol{n}\left(s_{i-1}^{\prime}\right), \delta \boldsymbol{x}\left(s_{i-1}^{\prime}, s_{i}^{\prime}\right)\right\rangle}{\left|\delta \boldsymbol{x}\left(s_{i-1}^{\prime}, s_{i}^{\prime}\right)\right|^{2}}\left[k \frac{\left\langle\boldsymbol{n}\left(s_{n}^{\prime}\right), \delta \boldsymbol{x}\left(s_{n}^{\prime}, s\right)\right\rangle\left\langle\boldsymbol{n}(s), \delta \boldsymbol{x}\left(s_{n}^{\prime}, s\right)\right\rangle}{4 r^{3}}\right. \\
& \left.-\mathrm{i} \frac{\left\langle\boldsymbol{n}\left(s_{n}^{\prime}\right), \boldsymbol{n}(s)\right\rangle}{2 r^{2}}\right]
\end{aligned}
$$

where $s^{\prime}=\left(s_{1}^{\prime}, \ldots, s_{n}^{\prime}\right), t=\left(t_{1}, \ldots, t_{n}\right)$ and we use the convention $s_{0}^{\prime}=s_{n+1}=s$. As in the discussion after (77) one sees that the main contributions to (85) come from orbit segments, starting in $s$ and returning to $s$ after $n$ reflections at the boundary. The total length of these orbits is $2 r+2 \sum_{i} t_{i}$ and since this expression appears as the argument of $\hat{\rho}$, the only orbit contributing to the integral (85) is the one with $s_{1}^{\prime}=s_{2}^{\prime}=\cdots=s_{n}^{\prime}=s$ and $t_{i}=r=0$, thanks to the small support of $\hat{\rho}$. So we can approximate $\left(\delta \boldsymbol{x}\left(s_{i-1}^{\prime}, s_{i}^{\prime}\right)\right)^{2} \approx\left(s_{i-1}^{\prime}-s_{i}^{\prime}\right)^{2}$ for all $i$ in the exponent of the integrand of (85), and in the prefactor $b$ we can set $s_{i}^{\prime}=s$ for all $i$. Now a substitution $s^{\prime} \rightarrow s^{\prime} / k, t \rightarrow t / k$ and $r \rightarrow r / k$ makes the integrand independent of $k$ up to a factor of $k^{-2 n}$, which together with the former prefactor $k^{n+1}$ gives an overall factor $k^{-n+1}$. A more careful analysis shows that the resulting integrals diverge at $r=0$, so we should use the regularization which we already applied to the computation of $g_{1}\left(k, s, s^{\prime}\right)$. But this does not change the final result

$$
g_{n}(k, s, s)=O\left(k^{1-n}\right) .
$$

\section{Appendix D. The completeness relation}

In this appendix we derive the completeness relation (54). By the results of appendix $\mathrm{C}$ we get

$\int_{\partial \Omega} \varphi\left(s^{\prime}\right) g^{\rho}\left(k, s, s^{\prime}\right) \mathrm{d} s^{\prime}=\int_{\partial \Omega} \varphi\left(s^{\prime}\right) g_{0}^{\rho}\left(k, s, s^{\prime}\right) \mathrm{d} s^{\prime}+\int_{\partial \Omega} \varphi\left(s^{\prime}\right) g_{1}^{\rho}\left(k, s, s^{\prime}\right) \mathrm{d} s^{\prime}+O(1 / k)$

if $\varphi \in C^{\infty}(\partial \Omega)$ and $\rho$ satisfies conditions (35). The first term on the right-hand side can easily be computed by using (72), the method of stationary phase, and by observing that due to the cut-off introduced by $\hat{\rho}$ the only stationary points come from $s^{\prime}=s$. The result is

$$
\int_{\partial \Omega} \varphi\left(s^{\prime}\right) g_{0}^{\rho}\left(k, s, s^{\prime}\right) \mathrm{d} s^{\prime}=\frac{2}{\pi} \varphi(s)+O(1 / k)
$$


where we have assumed that $a(z)=1 / z^{2}+O\left(1 / z^{4}\right)$, see (34). The computation of the second term in (88) is similar but more complicated. Using now (77) and solving the resulting $s^{\prime}$ and $s^{\prime \prime}$-integrals with the method of stationary phase leads to

$$
\begin{aligned}
\int_{\partial \Omega} \varphi\left(s^{\prime}\right) g_{1, \zeta}^{\rho}( & \left.k, s, s^{\prime}\right) \mathrm{d} s^{\prime}=\operatorname{Im}\left[C k^{3-\zeta} \kappa(s) \varphi(s) \mathrm{e}^{-\mathrm{i} \frac{\pi}{2}(\zeta+1)}\right. \\
& \left.\times \int_{0}^{\infty} \int_{0}^{\infty} \frac{t^{1 / 2}}{r^{5 / 2-\zeta}} \hat{\rho}(2(t+r)) \mathrm{e}^{\mathrm{i} k(t+r)} \mathrm{d} r \mathrm{~d} t\right](1+O(1 / k))
\end{aligned}
$$

where we have collected all factors not depending on $k$ and $s$ in the real constant $C$. Introducing new coordinates $v=t+r, w=t-r$ allows us to solve the integrals, and after setting $\zeta=1$ we arrive at

$$
\int_{\partial \Omega} \varphi\left(s^{\prime}\right) g_{1}^{\rho}\left(k, s, s^{\prime}\right) \mathrm{d} s^{\prime}=C^{\prime \prime} \frac{\kappa(s)}{k} \varphi(s)+O\left(1 / k^{2}\right)=O(1 / k)
$$

for $k \rightarrow \infty$, with another constant $C^{\prime \prime}$. Therefore result (54) is established.

\section{References}

[1] Stöckmann H-J 1999 Quantum Chaos (Cambridge: Cambridge University Press)

[2] Rellich F 1940 Darstellung der Eigenwerte von $\Delta u+\lambda u=0$ durch ein Randintegral Math. Z. 46 635-6

[3] Bäcker A and Schubert R 1999 Chaotic eigenfunctions in momentum space J. Phys. A: Math. Gen. 32 4795-815

[4] Bäcker A and Schubert R 2002 Autocorrelation function of eigenstates in chaotic and mixed systems J. Phys. A: Math. Gen. 35 539-64

[5] Tualle J M and Voros A 1995 Normal modes of billiards portrayed in the stellar (or nodal) representation Chaos, Solitons Fractals 5 1085-102

[6] Simonotti F P, Vergini E and Saraceno M 1997 Quantitative study of scars in the boundary section of the stadium billiard Phys. Rev. E 56 3859-67

[7] Hörmander L 1985 The Analysis of Linear Partial Differential Operators IV (Berlin: Springer)

[8] Bäcker A, Steiner F and Stifter P 1995 Spectral statistics in the quantized cardioid billiard Phys. Rev. E 52 2463-72

[9] Hörmander L 1985 The Analysis of Linear Partial Differential Operators III (Berlin: Springer)

[10] Robnik M 1983 Classical dynamics of a family of billiards with analytic boundaries J. Phys. A: Math. Gen. 16 3971-86

[11] Robnik M 1984 Quantising a generic family of billiards with analytic boundaries J. Phys. A: Math. Gen. 17 1049-74

[12] Bäcker A and Schubert R 2002 Amplitude distribution of eigenfunctions in mixed systems J. Phys. A: Math. Gen. 35 527-38

[13] Bäcker A, Schubert R and Stifter P 1998 Rate of quantum ergodicity in Euclidean billiards Phys. Rev. E 57 $5425-47$

Bäcker A, Schubert R and Stifter P 1998 Phys. Rev. E 585192 (erratum)

[14] Ozawa S 1993 Asymptotic property of eigenfunction of the Laplacian at the boundary Osaka J. Math. 30 $303-14$

[15] Berry M V and Wilkinson M 1984 Diabolical points in the spectra of triangles Proc. R. Soc. A 392 15-43

[16] Boasman P A 1994 Semiclassical accuracy for billiards Nonlinearity 7 485-537

[17] Balian R and Bloch C 1970 Distribution of eigenfrequencies for the wave equation in a finite domain I. Three-dimensional problem with smooth boundary surface Ann. Phys., NY 60 401-447

Balian R and Bloch C 1974 Ann. Phys., NY 84 559-63 (erratum)

[18] Dimassi M and Sjöstrand J 1999 Spectral Asymptotics in the Semi-Classical Limit (Cambridge: Cambridge University Press)

[19] Guenther R B and Lee J W 1996 Partial Differential Equations of Mathematical Physics and Integral Equations (Mineola, NY: Dover)

[20] Bunimovich L A 1974 On ergodic properties of certain billiards Funct. Anal. Appl. 8 254-5

[21] Bunimovich L A 1979 On the ergodic properties of nowhere dispersing billiards Commun. Math. Phys. 65 295-312

[22] Dullin H R and Bäcker A 2001 About ergodicity in the family of limaçon billiards Nonlinearity 14 1673-87 